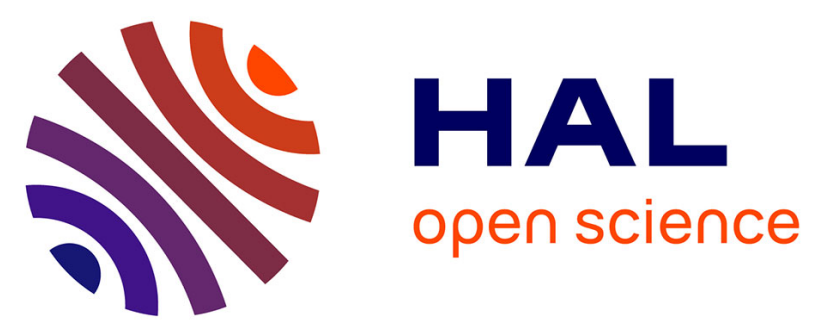

\title{
Characterization of rainbow trout gonad, brain and gill deep cDNA repertoires using a Roche 454-Titanium sequencing approach
}

Aurélie Le Cam, Julien Bobe, Olivier Bouchez, Cédric Cabeau, Olivier Kah, Christophe C. Klopp, Jean-Jacques Lareyre, Isabelle Leguen, Jérôme Lluch, Jérôme Montfort, et al.

\section{To cite this version:}

Aurélie Le Cam, Julien Bobe, Olivier Bouchez, Cédric Cabeau, Olivier Kah, et al.. Characterization of rainbow trout gonad, brain and gill deep cDNA repertoires using a Roche 454-Titanium sequencing approach. Gene, 2012, 500 (1), pp.32-39. 10.1016/j.gene.2012.03.053 . hal-01205069

\section{HAL Id: hal-01205069 \\ https://hal.science/hal-01205069}

Submitted on 29 May 2020

HAL is a multi-disciplinary open access archive for the deposit and dissemination of scientific research documents, whether they are published or not. The documents may come from teaching and research institutions in France or abroad, or from public or private research centers.
L'archive ouverte pluridisciplinaire HAL, est destinée au dépôt et à la diffusion de documents scientifiques de niveau recherche, publiés ou non, émanant des établissements d'enseignement et de recherche français ou étrangers, des laboratoires publics ou privés. 
1 Post-Print: Gene 2012500 (1), 32-39. DOI : 10.1016/j.gene.2012.03.053

3 Characterization of rainbow trout gonad, brain and gill deep cDNA

4 repertoires using a Roche 454-Titanium sequencing approach

5

6

$23{ }^{1}$ A.L.C. produced the double-stranded cDNA, supervised the construction and 24 sequencing of the 454 libraries, and participated in the writing of the manuscript.

${ }^{a}$ INRA, UR1037 LPGP Fish Physiology and Genomics, F-35000 Rennes, France.

${ }^{\mathrm{b}}$ INRA, Sigenae UR875 BIA, F-31326 Castanet-Tolosan Cedex, France.

${ }^{\mathrm{c}}$ UMR CNRS 6026, IFR 140, Université de Rennes 1, France.

${ }^{\mathrm{d}}$ INRA, Plateforme Génomique, Génopole Toulouse / Midi-Pyrénées, Auzeville, F-31326

Castanet-Tolosan, France.

${ }^{\S}$ Corresponding author: Tel.: 0033202234850 09, Fax: 003320223485020

E-mail address: yann.guiguen@rennes.inra.fr

Keywords: transcriptome; next-generation sequencing (NGS); fish; salmonids; rainbow trout.

\section{$-1-$}


${ }^{2}$ O.B. and J.L. aided in the construction of the 454 libraries and performed the sequencing reactions.

${ }^{3}$ J.M., C.C., F.M. and C.K. developed the rainbow trout contig browser and performed the bioinformatic analyses.

${ }^{4}$ J.B., B.N., O.K., A.S., I.L.G., P.P., J.J.L., and P.Y.R. analyzed the tissue-specific repertoires, performed QPCR analysis and drafted parts of the manuscript.

${ }^{5}$ Y.G. designed and supervised the study, participated in data analysis and drafted the final manuscript.

Abbreviations: ACTH, adrenocorticotropic hormone; amh, anti-müllerian hormone gene; AMP, adenosine monophosphate; bHLH, basic-helix-loop-helix; Blbp, Brain Lipid Binding protein; bp, base pair(s); Btg1, B Cell Translocation gene 1; cDNA, DNA complementary to RNA; CE, cornified envelope; dazl, deleted in azoospermia gene; DDD, Digital Differential Display; dmrt1, double-sex and mab-3 related transcription factor gene 1; EST, Expressed Sequenced Tag; fabp7a, fatty acid binding protein gene 7a; gap43, growth associated protein 43 gene; Gb, giga base; gdf9, growth and differentiation factor gene 9; glast, glutamate aspartate transporter gene; $g s d f$, gonadal soma-derived factor gene; Na/K-ATPase, sodium-potassium adenosine triphosphatase; nrnl, neuritin gene 1; neurodl, Neurogenic differentiation 1 gene; NGS, Next-Generation Sequencing; rec8, Meiotic recombination protein REC8 homolog; RNA-seq, RNA sequencing; ocpi, oocyte cysteine protease inhibitor gene; POMC, pro-opiomelanocortin; pou5f1, POU domain class V transcription factor 1 gene; RyR1, Ryanodine receptor 1; SAGE, Serial analysis of gene expression; Sox9a, SRY-box containing gene 9a; Smyd1, SRA, NCBI Sequence Read Archive; sycp 1, 2 and 3, synaptonemal complex protein; rhcg2, Rh type C glycoprotein2a 
gene; tudor1, tudor domain containing 1; TBX19, T-box transcription factor; tbx19,

51 T-box transcription factor gene 19; tubal, tubulin alpha1 gene; vasa, vasa homolog; wnt11, Wingless-type MMTV integration site family member 11; zarl, zygote arrest gene 1; ZP, zona pellucida; zpc1 and 5; zpax, egg envelope component ZPAX; zar1, zygote arrest gene 1.

\section{Abstract}

Rainbow trout, Oncorhynchus mykiss, is an important aquaculture species worldwide and, in addition to being of commercial interest, it is also a research model organism of considerable scientific importance. Because of the lack of a whole genome sequence in that species, transcriptomic analyses of this species have often been hindered. Using next-generation sequencing (NGS) technologies, we sought to fill these informational gaps. Here, using Roche 454-Titanium technology, we provide new tissue-specific cDNA repertoires from several rainbow trout tissues. Nonnormalized cDNA libraries were constructed from testis, ovary, brain and gill rainbow trout tissue samples, and these different libraries were sequenced in 10 separate halfruns of 454-Titanium. Overall, we produced a total of 3 million quality sequences with an average size of $328 \mathrm{bp}$, representing more than $1 \mathrm{~Gb}$ of expressed sequence information. These sequences have been combined with all publicly available rainbow trout sequences, resulting in a total of 242,187 clusters of putative transcript groups and 22,373 singletons. To identify the predominantly expressed genes in different tissues of interest, we developed a Digital Differential Display (DDD) approach. This

71 approach allowed us to characterize the genes that are predominantly expressed

72 within each tissue of interest. Of these genes, some were already known to be tissue-

73 specific, thereby validating our approach. Many others, however, were novel 
candidates, demonstrating the usefulness of our strategy and of such tissue-specific resources. This new sequence information, acquired using NGS 454-Titanium technology, deeply enriched our current knowledge of the expressed genes in rainbow trout through the identification of an increased number of tissue-specific sequences. This identification allowed a precise cDNA tissue repertoire to be characterized in several important rainbow trout tissues. The rainbow trout contig browser can be accessed at the following publicly available web site (http://www.sigenae.org/).

\section{Introduction}

Rainbow trout, Oncorhynchus mykiss, is an important aquaculture species worldwide and, in addition to being of commercial interest, it is also a model research organism of considerable scientific importance (reviewed by Thorgaard et al. (Thorgaard et al., 2002)). Many genomic resources are available for this species, including extensive classical Expressed Sequenced Tag (EST) repertoires (Rexroad et al., 2003; Govoroun et al., 2006; Koop et al., 2008); however, because of the lack of a currently available whole genome sequence in that species, genomic analysis has been inhibited by the limited knowledge of the rainbow trout transcriptome. The recent development of next-generation sequencing (NGS) has been a major technological breakthrough (Metzker, 2010). This technique provides new opportunities to better characterize gene repertoires, even in non-model species for which a whole genome sequence is unavailable (Crawford et al., 2010; Mizrachi et al., 2010; Fraser et al., 2011). These NGS technologies are now widely used for transcript expression profiling, and this approach, also referred to as RNA-seq, is progressively replacing classical techniques such as SAGE analysis or DNA microarrays (Costa et al., 2010). 
Using NGS and 454-Titanium technology, this study sought to complement the current knowledge of the rainbow trout transcriptome by providing new tissuespecific cDNA repertoires from different rainbow trout tissues. 454-Titanium technology has already been used to characterize rainbow trout transcripts (Salem et al., 2010); however, tissue-specific information is currently lacking. We thus developed sequencing resources and bioinformatics tools to initiate expression profiling analyses on the vast new collection of tissue-specific sequence information generated by this study. These first analyses demonstrate that our approach is reliable.

These new resources may now be utilized to search for more subtle genetic regulation events, allowing the identification of genes that are predominantly expressed under different physiological conditions.

\section{Materials and Methods}

\subsection{Tissue sampling and RNA extraction}

Total RNA was extracted from the collected tissues (gill, testis, brain and ovary) with TRIzol $^{\mathrm{TM}}$ reagent (Invitrogen, Cergy-Pontoise, France) according to the manufacturer's instructions. The total RNA yield was estimated using a Nanodrop 1000 spectrophotometer (Labtech, Palaiseau, France), and RNA quality was examined with an Agilent Bioanalyzer (Agilent Technologies, Massy, France). Only good quality total RNA samples (RNA Integrity Number, RIN > 8) were selected for further use. The total RNA from several extractions was pooled to minimize the impact of biological variations and to obtain the amount of RNA required for library preparation. Because of the presence of mucus, the gill sample pools were purified with the NucleoSpin ${ }^{\circledR}$ RNA II kit (Macherey-Nagel, Hoerdt, France) according to the "Clean-up of RNA from reaction mixtures" protocol. This purification was made from 

$200 \mu \mathrm{g}$ of the total RNA mix, and the elution was performed twice with $70 \mu \mathrm{lof} \mathrm{H}_{2} \mathrm{O}$

123 (2 successive elutions). mRNA was then isolated from $250 \mu \mathrm{g}$ of total RNA using the

124 Nucleotrap ${ }^{\circledR}$ mRNA kit (Macherey-Nagel, Hoerdt, France) as described in the manufacturer's manual, with the exception of the elution procedure. Here, $40 \mu 1$ of

126 pre-warmed $\left(68^{\circ} \mathrm{C}\right) \mathrm{H}_{2} \mathrm{O}$ were used per $40 \mu \mathrm{l}$ of Oligo(dT) latex beads. Three rounds of elution were performed. The quality of the mRNA and the depletion of ribosomal France).

\subsection{Double stranded-cDNA preparation}

131 Double-stranded cDNA was prepared as previously described (Leroux et al., 2010)

132 using modifications of the SuperScript Double-Stranded cDNA Synthesis Kit

133 (Invitrogen, Cergy-Pontoise, France) protocol. Briefly, we used a modified primer (5'

134 GAGAGAGAGACTGGAG(T)16VN 3') containing the GsuI restriction site and methylated dCTP (10 mM dm5CTP, Fermentas, Saint-Remy-les-Chevreuse, France) during the first-strand cDNA synthesis. The first strand was synthesized from $3 \mu \mathrm{g}$ of mRNA. The next steps (second strand synthesis, RNase I digestion, phenol/chloroform extraction and ethanol precipitation) were performed according to the manufacturer's instructions. The pellets were resuspended in $9 \mu 1$ of water. Three to five independent syntheses were made for each sample and pooled to obtain a total of 3.5 to $5 \mu \mathrm{g}$ of total cDNA. Next, the double-stranded cDNA was digested by GsuI (5 $\mathrm{U}$ ) for $90 \mathrm{~min}$ at $30^{\circ} \mathrm{C}$ to remove the polyA tail. The cDNA samples were quantified using the Quant-iT PicoGreen kit (Invitrogen, Cergy-Pontoise, France). 
146 After cDNA nebulization, the libraries were prepared using the GS DNA Library

147 Preparation Kit according to the manufacturer's recommendations (Roche Applied

148 Science, Meylan, France); 3.5 to $5 \mu \mathrm{g}$ of each cDNA sample were used to generate

149 the libraries. Sheared DNA was ligated to the linker for emPCR and sequencing. The

150 final libraries were quantified with the SlingShotTM kit using the Fluidigm ${ }^{\circledR}$ Digital

151 Array according to the manufacturer's instructions. Pyrosequencing using 454/Roche

152 GS FLX Titanium chemistry was performed with the GS Titanium LV emPCR and

153 sequencing kits according to the manufacturer's instructions. Each sample was

154 sequenced on one region of a two-region Picotiterplate.

155

156

\subsection{Real-time PCR analysis}

157 Real-time PCR was performed using the Step One Plus system (Applied Biosystems,

158 Foster City, USA) as previously described (Desvignes et al., 2009). For each tissue,

159 three separate reverse transcription reactions were conducted using $2 \mu \mathrm{g}$ of DNase-

160 treated RNA that originated from three different fishes. Control reactions were

161 performed in the absence of reverse transcriptase. RT products and control reaction

162 samples were diluted $1 / 25$, and $4 \mu 1$ was used for each real-time PCR reaction. Real-

163 time PCR was performed with the Fast SYBR Green Master Mix (Applied

164 Biosystem) and either 300 or $600 \mathrm{~nm}$ of each primer. All real-time PCR reactions

165 were performed in duplicate. After amplification, a melting curve was performed

166 according to the manufacturer recommendations to check the specificity of

167 amplification. The relative expression of each gene was normalized to the level of $18 \mathrm{~S}$

168 gene amplification and was calculated as the percentage of the highest expression 
169 level recorded for each gene. The sequences of the primers used are listed in

170 Supplementary file 2.

\subsection{In situ hybridization}

172 Digoxigenin-labeled antisense RNA probes were synthesized from a PCR-amplified

173 template (EST clone: BX085208 containing the rainbow trout $\beta$-enolase sequence)

174 using T3 RNA polymerase. In situ hybridizations were performed on transverse

175 sections of rainbow trout embryos as previously described (Gabillard et al., 2003),

176 with minor modifications.

177

178

179

180

181

182

183

184

185

186

187

188

189

190

191

192

\subsection{Bioinformatic analysis of the sequence data}

The initial sequence set used for this transcriptome assembly was composed of publicly available mRNA sequences from Genbank, Sanger ESTs from dbEST, GS-

Flx 454 RNA-Seq from SRA (including a complete rainbow trout muscle 454 dataset publicly available under the SRA accession number SRA029716) and the locally produced 454 Titanium RNA-Seq reads that are available under the SRA accession number SRA026493 (Table 1). The reads were cleaned to remove sequences composed of less than 100 base pairs, polyA tails, and E. coli and yeast contaminating sequences using SeqClean (http://compbio.dfci.harvard.edu/tgi/software/). To ease the assembly process, our next step was to remove redundancy using the cd-hit-est software (Li and Godzik, 2006). This software discarded those reads that were included in other reads. A threshold of 0.98 was chosen, producing a non-redundant representative read set (parameters -M 30000 -d 0 -n 10 -1 11 -r 1 -p 1 -g 1 -G 1 -c 0.98 -aS $0.5-\mathrm{T} 8$ ). The discarded reads were repositioned in the resultant contigs after assembly using the inclusion coordinates. The assembly was performed using TGICL (Pertea et al., 2003), which first clusters the reads using megablast, given a similarity 
193 threshold (-p 96) and a similarity length (-1 60), and then, using the mRNAs as

194 backbones, splits the clusters larger than a given size (-s 100000). The program then

195 produces alignment files and consensus sequences with CAP3. All of the 454 singlets

196 were removed from the final results, which contained 219,814 contigs and 22,373

197 singlets. The contigs and singlets were annotated by similarity searches using a large

198 set of nucleotide and protein reference databases, including UniProtKB/Swiss-Prot,

199 RefSeq protein, RefSeq RNA, PFAM, DFCI gene indices, UniGene clusters and

200 Ensembl transcripts for numerous species. The full list of databases is available on the

201 home page of the assembly (http://public-

202 contigbrowser.sigenae.org:9090/Oncorhynchus_mykiss/index.html). The e-value

203 thresholds that were used to determine similarities were dependent upon the database

204 that was used; $\mathrm{e}^{-10}$ was used for transcript databases, $\mathrm{e}^{-2}$ was used for species-specific

205 nucleic databases, and $\mathrm{e}^{-5}$ was used for all others. The UniProtKB/Swiss-Prot

206 similarity search was performed using a bi-directional best hit approach to identify

207 putative orthologs, and the resulting annotation character string had a $[\mathrm{BBH}]$ tag.

208 Variation detection was also performed using the contigs. The in-house detection

209 script screens the CAP3 alignment files to find substitutions that occur at least twice

210 at a position with a depth of at least 6 sequences and that have no other substitution in

211 a twenty base pair window around this position. All of the resulting files from the

212 assembly, the annotation and the variation detection steps were then reformatted to

213 populate the contig browser database, which was used to show the contig layout, and

214 the BioMart (Haider et al., 2009) database, which enables a large variety of queries.

215 The contig browser was built using the Ensembl 40 web code (Birney et al., 2006)

216 and provides such new features as the Digital Differential Display view and the Venn 
217 diagram view. The contig and singlet FASTA files and the BioMart tabular files can

218 be downloaded from the web server.

\section{Results and Discussion}

\subsection{The generation of tissue-specific transcriptomic resources using a RNA-}

\section{Seq approach}

223 Eight non-normalized cDNA libraries were constructed from rainbow trout testis,

224 ovary, brain, and gill tissue samples and were subsequently sequenced in 10 separate

225 half-runs using 454-Titanium (Table 1). Overall, we produced a total of 3 million

226 good-quality sequences (testis, 1,234,260; ovary, 738,510; brain, 481,363; and gill,

227613,223 ) with an average sequence size of $328 \mathrm{bp}$. Collectively, these sequences

228 constitute more than $1 \mathrm{~Gb}$ of expressed sequence information. This raised the total

229 number of expressed sequences that are publicly available for rainbow trout to 4.8

230 millions. In contrast to previous large NGS datasets produced in the rainbow trout

231 (Salem et al., 2010), our strategy also provided tissue-specific information that was

232 not previously available for this species. All of the acquired sequences were deposited

233 in the NCBI Sequence Read Archive (SRA) and were assembled along with the

234 publicly available rainbow trout sequences. This resulted in a total of 242,187 clusters

235 of putative transcripts (contigs) and 22,373 singletons. These sequences and their

236 assembly can be accessed at the following publicly available web site

237 (http://www.sigenae.org/). This browser is based on an Ensembl architecture

238 (Hubbard et al., 2002; Stabenau et al., 2004). One can utilize many classical features

239 to browse all of the computed information on each contig and can extract large user-

240 specific datasets using the Biomart generic interface (Smedley et al., 2009). In silico

$$
-10-
$$

\section{Comment citer ce document}


241 strategies have often been used to mine sequence databases and discover new genes

242 with a predominant expression pattern in one tissue or a group of tissues (Murray et

243 al., 2007); however, the use of such strategies has been restricted to species with large

244 available sequence datasets. In non-model species, this approach has, in contrast,

245 often been limited by the rather low number of sequences available. With the recent

246 advent of NGS, this limitation is no longer critical. Information on many non-model

247 species is now more accessible due to RNA-Seq approaches (Wilhelm and Landry,

248 2009), allowing for the characterization of genes with differential expression patterns

249 (Crawford et al., 2010; Mizrachi et al., 2010; Fraser et al., 2011). Therefore, we

250 applied this approach to our own dataset to validate its utility.

\subsection{The use of the 454 tissue-specific transcriptomic resource for the} identification of tissue-predominant transcripts

253 A Digital Differential Display (DDD) analysis tool was implemented within the

254 rainbow trout sequence browser to characterize contigs with a predominant expression 255 pattern. DDD analysis was subsequently performed using the 454 sequencing data 256 that was obtained from this study. For each tissue of interest (ovary, testis, brain, gill 257 and muscle), we compared the number of reads contained in each of the contigs in the 258 tissue of interest to all of the remaining tissue libraries. Some of these examples are 259 discussed below, and the DDD software is available within the rainbow trout contig 260 browser to allow for further user-specific queries. To confirm that this strategy is 261 effective for the identification of genes that are predominantly expressed in a given 262 tissue, we carried out qPCR validation for 9 genes (see Fig 1 and Tables 2 to 6).

$263 \quad 3.2 .1$ The identification of genes that are preferentially expressed in the rainbow trout 264 ovary 
265 When performing a DDD analysis on the trout ovarian samples using a 10-fold cut-

266 off, 614 contigs were found to be over abundant in the ovarian/oocyte libraries in

267 comparison to the non-ovarian libraries. Among those 614 contigs, 438 contigs were

268 found only 1 or 2 times in the non-ovarian libraries (see additional file 1). In addition,

269432 contigs were found at least 10 times in the ovarian libraries but were never

270 detected in the non-ovarian libraries. Among the most overrepresented genes found in

271 the ovarian libraries, we identified several ZP domain-containing genes. Our results

272 show that, in agreement with existing data from medaka (Oryzias latipes) (Kanamori

273 et al., 2003), the $z p c 1, z p c 5$, and zpax genes are predominantly expressed in the

274 ovaries in the rainbow trout (Table 2). Similarly, several genes that are known to be

275 specifically or predominantly expressed in rainbow trout oocytes or ovaries were

276 identified. In agreement with existing data on rainbow trout (Bobe et al., 2008),

277 zygote arrest 1 (zarl) and growth and differentiation factor 9 ( $g d f 9$ ) exhibited a very

278 strict ovarian-predominant profile. Similarly, oocyte cysteine protease inhibitor (ocpi)

279 was dramatically overexpressed in the ovarian libraries, again in agreement with

280 existing data (Bobe and Goetz, 2001). In addition to these previously documented

281 patterns, which validate our approach, the DDD analysis has led to the identification

282 of many genes that also putatively exhibit predominant or specific ovarian and/or

283 oocyte expression. Interestingly, our data also revealed the predominant expression of

284 several ovarian genes in the rainbow trout that are known to be strongly expressed in

285 the zebrafish (Danio rerio) oocyte, such as bucky ball (Marlow and Mullins, 2008), or

286 in the medaka, such as the transcription factor pou5f1 (Sanchez-Sanchez et al., 2010)

287 (Fig. 1 and Table 2). Furthermore, we have shown the ovarian-predominant

288 expression of wntl1 (Fig. 1 and Table 2). In sexually mature rainbow trout, wnt11

289 mRNA was detected in all assayed tissues. In comparison to skin, which had the 

wnt11 in post-ovulatory ovaries and a 1,700-fold higher expression in oocytes (Fig. 1). Our results are consistent with previous studies reporting the expression of wnt11 in mouse granulosa cells and oocytes (Harwood et al., 2008). Interestingly, wnt11 is a maternal transcript. Maternal wnt11 mRNA has been shown to accumulate in Xenopus oocytes (Ku and Melton, 1993), where it plays a role in axis formation in the embryo (Tao et al., 2005). Together, these results suggest the participation of maternally inherited wnt 11 mRNA in teleost early development. The DDD analysis also allowed the identification of novel oocyte-predominant genes in rainbow trout

299 (see Additional File 1). Such genes deserve further attention.

301 When performing a DDD analysis of the trout testicular samples, we characterized 302503 contigs that were only expressed in the testes, with a minimum of 10 reads used 303 as a threshold. In addition, 590 contigs were significantly overexpressed in the testes, 304 with a 10-fold minimum change and a threshold of 10 reads (see additional file 1 ). As 305 expected, numerous contigs corresponded to transcripts that are known to be 306 preferentially expressed in the testes of several vertebrate species, including the 307 rainbow trout (Rolland et al., 2009). These transcripts are involved in the sexual 308 differentiation of the male gonad (Sox9a, dmrt1, amh) and in specific testicular 309 functions such as the regulation of germ cell proliferation ( $a m h, g s d f)$, meiosis (sycpl, 310 sycp2 and sycp3, rec8, dazl, vasa, tudor1), and spermiogenesis (Dynein intermediate 311 chain 1, microtubule associated protein 6). These observations illustrate the reliability 312 of the DDD method in the analysis of the 454 sequencing data. Interestingly, our 313 analysis revealed several transcripts that were unexpectedly overexpressed in trout 314 testes. For instance, one contig sequence encodes the trout counterpart of the 
316 of a multigene superfamily that harbors a conserved DNA binding domain called the

317 T-box (see (Plageman and Yutzey, 2005) for review). The TBX transcription factors

318 are involved in the regulation of developmental processes. In humans and mice,

319 TBX19 expression is restricted to two pituitary cell types that express pro-

320 opiomelanocortin (POMC). In trout, enhanced TBX19 expression was also detected in

321 the pituitary along with the testes (Fig. 1). A mutation of the $t b x 19$ gene in humans

322 results in the impaired differentiation of the POMC cell lineages as well as ACTH

323 deficiency (Vallette-Kasic et al., 2007). An understanding of the biological relevance

324 of the enhanced expression of the $t b x 19$ gene in trout testes will require further

325 investigation. We also noted that several transcripts that originated from transposable

326 elements were among the most frequently detected transcripts in trout testes. These

327 transcripts encode reverse transcriptases, transposases and partial envelope proteins

328 (Table 3). Transposable elements have long been considered "junk" DNA, but there is

329 accumulating evidence that they may have been recruited by the progenote to fulfill

330 important biological functions(Volff, 2006, Kleene et al., 1998). For instance,

331 retroviral envelope proteins are required for the cell contact and fusion of human

332 trophoblast cells (Vargas et al., 2009). Moreover, the transposition of active mobile

333 elements in the vicinity of any gene can modulate gene expression, and it has been

334 proposed that these events may participate in the epigenetic regulation of genes in

335 somatic cells (see (Muotri et al., 2007, Collier and Largaespada, 2007) for reviews).

336 In addition, transposition is likely in germ cells, and this could be considered a major

337 source of genetic variation that can be passed on to further generations. The

338 identification of mobile transposable elements in the testes contributes to our

339 understanding of the mechanisms that lead to dynamic changes in the genome 
340 structure and may provide an explanation for the observed differences in the

341 reproductive capacity of individuals.

$342 \quad 3.2 .3$ The identification of genes that are preferentially expressed in muscle

343 When performing a DDD analysis for the trout muscle samples using a 10-fold cut-

344 off, 393 contigs were found to be overabundant in the white muscle libraries in

345 comparison to the non-muscle libraries (see Additional File 1). Among these, 266

346 contigs were found only 1 or 2 times in the non-muscle libraries. Of the top ten

347 overabundant contigs (exhibiting overexpression ranging from 2517- to 793-fold), six

348 were found to encode sarcomeric proteins such as myosins and troponins (Table 4).

349 Other sarcomeric protein-encoding genes were found to be overexpressed in the

350 muscle libraries including Lim domain-binding protein 3, nebulin and myozenin-1.

351 Additionally, sarcosin (kbtbd10, for Kelch repeat and BTB domain-containing protein

352 10, see Fig. 1), which is involved in the assembly of myofibrils (Greenberg et al.,

353 2008), and Stars, an actin-binding Rho-activating protein that promotes actin

354 polymerization (Arai et al., 2002), were predominantly expressed in the muscle

355 library. The DDD analysis is also strongly validated by the observation that most of

356 the enzymes identified in the muscle libraries were known to be muscle-specific

357 isoforms or enzymes. Among these enzymes were creatine kinase, Phosphoglycerate

358 mutase (pgam2) (see Fig. 1), Adenylate kinase isoenzyme 1, L-lactate dehydrogenase

359 A, Fructose-bisphosphate aldolase A, 6-phosphofructokinase, Glycogen

360 phosphorylase, AMP deaminase 1 and $\beta$-enolase (Table 4). Consistently, the in situ

361 hybridization of $\beta$-enolase revealed a strong hybridization signal specifically in

362 glycolytic white fast muscle fibers (Fig. 2). In agreement with existing data, the

363 histone methyl transferase Smyd1 (Tan et al., 2006) and the antiproliferative protein

364 Btg1 (Busson et al., 2005), which both regulate muscle differentiation, were clearly 
overexpressed in the muscle libraries. In addition, DDD analysis has led to the

366 identification of the genes encoding Ryanodine receptor 1 (RyR1) and calsequestrin,

367 which are known to participate in the formation of a complex that is required for $\mathrm{Ca}^{2+}$

368 ion release from the sarcoplasmic reticulum. This is an essential step in muscle

369 contraction (Zhang et al., 1997).

$370 \quad 3.2 .4$ The identification of genes that are preferentially expressed in the brain

371 When performing a DDD analysis with a 10-fold cut-off in the trout brain samples

372 that were taken 40 days post-fertilization, 313 contigs were found to be overabundant

373 in the brain libraries in comparison to the non-brain libraries. In addition, 365 contigs

374 were found at least 10 times in the brain libraries but were never detected in the non-

375 brain libraries. At the stage examined, the brain is organized and regionalized, but it is

376 still actively growing. Accordingly, many of the genes that were identified in the

377 brain samples are known to be involved in neurogenesis and/or neuronal

378 differentiation (Table 5). Some of these genes ( glast and fabp7a) are likely to be

379 expressed in the radial glial cells that are the main progenitors at this stage, whereas

380 others are involved in neuronal growth or neuron differentiation (synapsin,

381 neuromodulin, neuritin, and neuroD). Among the genes expected to be strongly

382 expressed in the developing brain is fabp $7 a$, and Brain Lipid Binding Protein (Blbp,

383 see Fig. 1). fabp $7 a$, is a member of the fatty acid-binding protein family that was

384 retrieved several times. This finding is of particular interest because, in mice, Blbp is

385 a recognized marker of radial glial cells, which are known to be progenitor cells in the

386 developing mammalian brain (Anthony and Heintz, 2008). Recently, it was shown

387 that blbp is also expressed in the brains of larval and adult fish (Diotel et al., 2010;

388 Marz et al., 2010). Ependymin (epdl) is a meningeal-derived extracellular matrix

389 glycoprotein that was also overrepresented in the brain in comparison to other tissues. 
390 Little is known about the function of such proteins; however, they are thought to

391 increase in abundance during periods of neuronal plasticity in mammals (Rother et al.,

392 1995). The hypothesis that ependymin is also involved in neurogenesis in fish is of

393 particular interest and will need to be further examined. The gene slcla2b encodes a

394 glial high-affinity glutamate transporter that was previously identified in a screen of

395 the zebrafish hypothalamus (Toro et al., 2009). In mammals, these glutamate

396 transporters are strongly expressed in astrocytes. In these cells, the excess of

397 glutamate is quickly removed from the extracellular space, where the glutamate level

398 is kept low. Such transporters are also expressed in the radial glial cells of rodents

399 and, likely, of fishes, although the latter has not been documented. Neuromodulin

400 (gap43) was also found to be expressed only in the brain (see Fig. 1), in agreement

401 with its recognized functions as a 'plasticity' protein that is highly expressed in

402 neuronal growth cones during development (Udvadia et al., 2001). Neurogenic

403 differentiation 1 (neurod1) is a member of the NeuroD family of basic-helix-loop-

404 helix (bHLH) transcription factors that are involved in brain differentiation and

405 patterning. Tubulin alpha chains (tubal) were also highly represented, which was

406 expected based on their known roles in neurotubule formation (Hieber et al., 1998).

407 Neuritin and synapsin, respectively, are involved in neurite growth and synapse

408 formation. Neuritin ( $n r n l)$ is expressed in the postmitotic neurons of the developing

409 nervous system and in neuronal structures that are undergoing plasticity in adults

410 (Naeve et al., 1997). Interestingly, neuritin was also represented in the libraries

411 constructed from non-nervous tissues. Synapsins are a family of proteins that are

412 implicated in the regulation of neurotransmitter release at synapses. Recent data

413 suggest that synapsins are not essential for synaptic function, but instead serve

414 important modulatory functions at the synapse (Kile et al., 2010). 
416 When comparing gill with other tissues using a DDD analysis with a 10 fold cut-off,

417 we found 313 contigs that were overrepresented in the gill libraries. Moreover, 365

418 contigs were only expressed in the gill libraries (see Additional File 1). As expected,

419 several of these genes are known to be specifically or predominantly expressed in the

420 gills (Table 6). Included in the gill-specific genes is a new calpain, which was

421 reported to be expressed in the trout gill by Salem and coworkers (Salem et al., 2006).

422 Additionally, we identified several ion transporters such as Na/K-ATPase $\alpha 1 \mathrm{a}$, which

423 was previously shown to be overexpressed in gill tissue by Richards et al. (Richards et

424 al., 2003). Proteins that are known to be regulators of cellular ion transport, such as

425 the FXYD proteins that are known to regulate the substrate affinity of the $\mathrm{Na} / \mathrm{K}$ -

426 ATPase enzyme (Garty and Karlish, 2006), were also overexpressed in gill tissue. In

427 the Atlantic salmon (Salmo salar), the expression of FXYD11 isoform was reported

428 to be 1,000-fold higher in gill than in other tissues (Tipsmark, 2008). In agreement

429 with this finding, our data revealed that this FXYD11 isoform is overexpressed in gill

430 tissue. Other interesting candidate genes that were found are known to be linked to

431 ammonia excretion. Ammonia is predominantly excreted in the gills in teleost fish.

432 Consequently, it is not surprising to find a higher expression of genes encoding

433 ammonia transporters (Rhesus ( $\mathrm{Rh}$ ) proteins) in gill tissue than in other organs. For

434 instance, we observed the predominant expression of Rh type $\mathrm{C}$ glycoprotein (rhcg2)

435 in the trout gill, in agreement with data reported by (Nawata et al., 2007). DDD

436 analysis also allowed us to identify new putative genes that are predominantly

437 expressed in gill tissue in comparison to other tissues. These include the zymogen

438 granule protein 16 ( $\mathrm{zg} 16)$ (see Fig. 1), which is considered to be a secretory lectin that

439 is involved in the regulation of granule secretion (Zhou et al., 2007), and the 
envoplakin (evpl) and corniferin genes, which are known in mammals to be involved

441 in maintaining the structure of the epithelium. Envoplakin (see Fig. 1) is a component

442 of desmosomes and cornified envelope (CE) and is expressed in keratinizing and non-

443 keratinizing squamous epithelia (Ruhrberg et al., 1996). Cornifelin is also a

444 component of the CE (Michibata et al., 2004). The cornified envelope provides a

445 protective barrier between the environment and the living layers of the skin and is

446 thought to play an important role in the maintenance of the integrity of the epidermis

447 in mammals. The presence of several CE proteins in gill tissue could suggest their

448 importance in maintaining the barrier that protects the gill epithelium from the water

449 environment.

\section{Conclusions}

The present work utilized 454-Titanium technology to uncover tissue-specific cDNA repertoires from various rainbow trout tissues. This important information has already allowed us to develop an in silico data mining program that searches for genes that are differentially expressed between tissues; however, these analyses are still difficult because of the inherent complexity of the de novo assembly of these large transcript sequencing datasets. This complexity has been already described (Costa et al., 2010)

457 and involves, for instance, the presence of different splice forms of genes, as well as 458 of allele-specific variants, which can split different contigs after the assembly process.

459 The current lack of a whole genome reference sequence in rainbow trout is a major 460 problem; however, ongoing projects should soon provide this genome resource, 461 allowing researchers to directly map these short read sequences onto the genome, 462 partially solving the difficulties inherent in de novo transcriptome assembly. 


\section{References}

465

466

467

468

469

470

471

472

473

474

475

476

477

478

479

480

481

482

483

484

485

486

487

Anthony, T.E. and Heintz, N. Genetic lineage tracing defines distinct neurogenic and gliogenic stages of ventral telencephalic radial glial development. Neural development 3 (2008), p. 30.

Arai, A., Spencer, J.A. and Olson, E.N. STARS, a striated muscle activator of Rho signaling and serum response factor-dependent transcription. The Journal of biological chemistry 277 (2002), pp. 24453-9.

Birney, E., Andrews, D., Caccamo, M., Chen, Y., Clarke, L., Coates, G., Cox, T., Cunningham, F., Curwen, V., Cutts, T., Down, T., Durbin, R., FernandezSuarez, X.M., Flicek, P., Graf, S., Hammond, M., Herrero, J., Howe, K., Iyer, V., Jekosch, K., Kahari, A., Kasprzyk, A., Keefe, D., Kokocinski, F., Kulesha, E., London, D., Longden, I., Melsopp, C., Meidl, P., Overduin, B., Parker, A., Proctor, G., Prlic, A., Rae, M., Rios, D., Redmond, S., Schuster, M., Sealy, I., Searle, S., Severin, J., Slater, G., Smedley, D., Smith, J., Stabenau, A., Stalker, J., Trevanion, S., Ureta-Vidal, A., Vogel, J., White, S., Woodwark, C. and Hubbard, T.J. Ensembl 2006. Nucleic Acids Research 34 (2006), pp. D556-61.

Bobe, J. and Goetz, F.W. Cysteine protease inhibitor is specifically expressed in preand early-vitellogenic oocytes from the brook trout periovulatory ovary. Mol Reprod Dev 60 (2001), pp. 312-8.

Bobe, J., Nguyen, T., Mahe, S. and Monget, P. In silico identification and molecular characterization of genes predominantly expressed in the fish oocyte. BMC Genomics 9 (2008), p. 499.

Busson, M., Carazo, A., Seyer, P., Grandemange, S., Casas, F., Pessemesse, L., Rouault, J.P., Wrutniak-Cabello, C. and Cabello, G. Coactivation of nuclear 
receptors and myogenic factors induces the major BTG1 influence on muscle differentiation. Oncogene 24 (2005), pp. 1698-710.

490

Collier, L.S. and Largaespada, D.A. Transposable elements and the dynamic somatic genome. Genome biology 8 Suppl 1 (2007), p. S5.

Costa, V., Angelini, C., De, F.I. and Ciccodicola, A. Uncovering the complexity of transcriptomes with RNA-Seq. J Biomed Biotechnol 2010 (2010), p. 853916.

Crawford, J.E., Guelbeogo, W.M., Sanou, A., Traore, A., Vernick, K.D., Sagnon, N. and Lazzaro, B.P. De novo transcriptome sequencing in Anopheles funestus using Illumina RNA-seq technology. PLoS One 5 (2010), p. e14202.

Desvignes, T., Pontarotti, P., Fauvel, C. and Bobe, J. Nme protein family evolutionary history, a vertebrate perspective. BMC Evol Biol 9 (2009), p. 256.

Diotel, N., Le Page, Y., Mouriec, K., Tong, S.K., Pellegrini, E., Vaillant, C., Anglade, I., Brion, F., Pakdel, F., Chung, B.C. and Kah, O. Aromatase in the brain of teleost fish: expression, regulation and putative functions. Frontiers in neuroendocrinology 31 (2010), pp. 172-92.

Fraser, B.A., Weadick, C.J., Janowitz, I., Rodd, H. and Hughes, K.A. Sequencing and characterization of the guppy (Poecilia reticulata) transcriptome. BMC Genomics 12 (2011), p. 202.

Gabillard, J.C., Duval, H., Cauty, C., Rescan, P.Y., Weil, C. and Le Bail, P.Y. Differential expression of the two GH genes during embryonic development of rainbow trout Oncorhynchus mykiss in relation with the IGFs system. Molecular Reproduction and Development 64 (2003), pp. 32-40.

Garty, H. and Karlish, S.J. Role of FXYD proteins in ion transport. Annual review of physiology 68 (2006), pp. 431-59. 
512 Govoroun, M., Le Gac, F. and Guiguen, Y. Generation of a large scale repertoire of Expressed Sequence Tags (ESTs) from normalised rainbow trout cDNA libraries. BMC.Genomics 7 (2006), p. 196.

515 Greenberg, C.C., Connelly, P.S., Daniels, M.P. and Horowits, R. Krp1 (Sarcosin) promotes lateral fusion of myofibril assembly intermediates in cultured mouse

Haider, S., Ballester, B., Smedley, D., Zhang, J., Rice, P. and Kasprzyk, A. BioMart Central Portal--unified access to biological data. Nucleic Acids Research 37 (2009), pp. W23-7.

Harwood, B.N., Cross, S.K., Radford, E.E., Haac, B.E. and De Vries, W.N. Members of the WNT signaling pathways are widely expressed in mouse ovaries, oocytes, and cleavage stage embryos. Developmental dynamics : an official publication of the American Association of Anatomists 237 (2008), pp. 1099-

Hieber, V., Dai, X., Foreman, M. and Goldman, D. Induction of alpha1-tubulin gene expression during development and regeneration of the fish central nervous system. Journal of neurobiology 37 (1998), pp. 429-40.

Hubbard, T., Barker, D., Birney, E., Cameron, G., Chen, Y., Clark, L., Cox, T., Cuff, Huminiecki, L., Kasprzyk, A., Lehvaslaiho, H., Lijnzaad, P., Melsopp, C., Mongin, E., Pettett, R., Pocock, M., Potter, S., Rust, A., Schmidt, E., Searle, S., Slater, G., Smith, J., Spooner, W., Stabenau, A., Stalker, J., Stupka, E., Ureta-Vidal, A., Vastrik, I. and Clamp, M. The Ensembl genome database project. Nucleic Acids Research 30 (2002), pp. 38-41. 
Kanamori, A., Naruse, K., Mitani, H., Shima, A. and Hori, H. Genomic organization of $\mathrm{ZP}$ domain containing egg envelope genes in medaka (Oryzias latipes). Gene 305 (2003), pp. 35-45.

Kile, B.M., Guillot, T.S., Venton, B.J., Wetsel, W.C., Augustine, G.J. and Wightman, R.M. Synapsins differentially control dopamine and serotonin release. The Journal of neuroscience : the official journal of the Society for Neuroscience 30 (2010), pp. 9762-70.

Kleene, K.C., Mulligan, E., Steiger, D., Donohue, K. and Mastrangelo, M.A. The mouse gene encoding the testis-specific isoform of Poly(A) binding protein (Pabp2) is an expressed retroposon: intimations that gene expression in spermatogenic cells facilitates the creation of new genes. Journal of Molecular Evolution 47 (1998), pp. 275-81.

Koop, B.F., von Schalburg, K.R., Leong, J., Walker, N., Lieph, R., Cooper, G.A., Robb, A., Beetz-Sargent, M., Holt, R.A., Moore, R., Brahmbhatt, S., Rosner, J., Rexroad, C.E., 3rd, McGowan, C.R. and Davidson, W.S. A salmonid EST genomic study: genes, duplications, phylogeny and microarrays. BMC Genomics 9 (2008), p. 545.

Ku, M. and Melton, D.A. Xwnt-11: a maternally expressed Xenopus wnt gene. Development 119 (1993), pp. 1161-1173.

Leroux, S., Feve, K., Vignoles, F., Bouchez, O., Klopp, C., Noirot, C., Gourichon, D., Richard, S., Leterrier, C., Beaumont, C., Minvielle, F., Vignal, A. and Pitel, F. Non PCR-amplified Transcripts and AFLP fragments as reduced representations of the quail genome for 454 Titanium sequencing. BMC research notes 3 (2010), p. 214. 
Marlow, F.L. and Mullins, M.C. Bucky ball functions in Balbiani body assembly and animal-vegetal polarity in the oocyte and follicle cell layer in zebrafish. Developmental Biology 321 (2008), pp. 40-50.

Marz, M., Chapouton, P., Diotel, N., Vaillant, C., Hesl, B., Takamiya, M., Lam, C.S., Kah, O., Bally-Cuif, L. and Strahle, U. Heterogeneity in progenitor cell subtypes in the ventricular zone of the zebrafish adult telencephalon. Glia $\mathbf{5 8}$ (2010), pp. 870-88.

Metzker, M.L. Sequencing technologies - the next generation. Nature reviews. Genetics 11 (2010), pp. 31-46.

Michibata, H., Chiba, H., Wakimoto, K., Seishima, M., Kawasaki, S., Okubo, K., Mitsui, H., Torii, H. and Imai, Y. Identification and characterization of a novel component of the cornified envelope, cornifelin. Biochemical and Biophysical Research Communications 318 (2004), pp. 803-13.

Mizrachi, E., Hefer, C.A., Ranik, M., Joubert, F. and Myburg, A.A. De novo assembled expressed gene catalog of a fast-growing Eucalyptus tree produced by Illumina mRNA-Seq. BMC Genomics 11 (2010), p. 681.

Muotri, A.R., Marchetto, M.C., Coufal, N.G. and Gage, F.H. The necessary junk: new functions for transposable elements. Human Molecular Genetics 16 Spec No. 2 (2007), pp. R159-67.

Murray, D., Doran, P., MacMathuna, P. and Moss, A.C. In silico gene expression analysis--an overview. Molecular cancer 6 (2007), p. 50.

Naeve, G.S., Ramakrishnan, M., Kramer, R., Hevroni, D., Citri, Y. and Theill, L.E. Neuritin: a gene induced by neural activity and neurotrophins that promotes neuritogenesis. Proceedings of the National Academy of Sciences of the United States of America 94 (1997), pp. 2648-53. 

Ammonia excretion in rainbow trout (Oncorhynchus mykiss): evidence for $\mathrm{Rh}$ glycoprotein and H+-ATPase involvement. Physiological genomics 31 (2007), pp. 463-74.

Pertea, G., Huang, X., Liang, F., Antonescu, V., Sultana, R., Karamycheva, S., Lee, Y., White, J., Cheung, F., Parvizi, B., Tsai, J. and Quackenbush, J. TIGR Gene Indices clustering tools (TGICL): a software system for fast clustering of large EST datasets. Bioinformatics 19 (2003), pp. 651-2.

Plageman, T.F., Jr. and Yutzey, K.E. T-box genes and heart development: putting the "T" in heart. Developmental dynamics : an official publication of the American Association of Anatomists 232 (2005), pp. 11-20.

Rexroad, C.E., Lee, Y., Keele, J.W., Karamycheva, S., Brown, G., Koop, B., Gahr, S.A., Palti, Y. and Quackenbush, J. Sequence analysis of a rainbow trout cDNA library and creation of a gene index. Cytogenetic and Genome Research 102 (2003), pp. 347-354.

Richards, J.G., Semple, J.W., Bystriansky, J.S. and Schulte, P.M. Na+/K+-ATPase alpha-isoform switching in gills of rainbow trout (Oncorhynchus mykiss) during salinity transfer. The Journal of experimental biology 206 (2003), pp. 4475-86.

Rolland, A.D., Lareyre, J.J., Goupil, A.S., Montfort, J., Ricordel, M.J., Esquerre, D., Hugot, K., Houlgatte, R., Chalmel, F. and Le Gac, F. Expression profiling of rainbow trout testis development identifies evolutionary conserved genes involved in spermatogenesis. BMC Genomics 10 (2009), p. 546. 
608 Rother, S., Schmidt, R., Brysch, W. and Schlingensiepen, K.H. Learning-induced expression of meningeal ependymin mRNA and demonstration of ependymin in neurons and glial cells. Journal of neurochemistry 65 (1995), pp. 1456-64.

Ruhrberg, C., Hajibagheri, M.A., Simon, M., Dooley, T.P. and Watt, F.M. Envoplakin, a novel precursor of the cornified envelope that has homology to

Salem, M., Rexroad, C.E., 3rd, Wang, J., Thorgaard, G.H. and Yao, J. desmoplakin. The Journal of cell biology 134 (1996), pp. 715-29. Characterization of the rainbow trout transcriptome using Sanger and 454pyrosequencing approaches. BMC Genomics 11 (2010), p. 564.

Salem, M., Rexroad, C.E. and Yao, J. Identification of a novel gill-specific calpain from rainbow trout (Oncorhynchus mykiss). Fish Physiology and Biochemistry 32 (2006), pp. 1-6.

Sanchez-Sanchez, A.V., Camp, E., Garcia-Espana, A., Leal-Tassias, A. and Mullor, J.L. Medaka Oct4 is expressed during early embryo development, and in primordial germ cells and adult gonads. Developmental dynamics : an official publication of the American Association of Anatomists 239 (2010), pp. 672-9.

Stabenau, A., McVicker, G., Melsopp, C., Proctor, G., Clamp, M. and Birney, E. The Kasprzyk, A. BioMart--biological queries made easy. BMC Genomics 10

629 Tan, X., Rotllant, J., Li, H., De Deyne, P. and Du, S.J. SmyD1, a histone 630 methyltransferase, is required for myofibril organization and muscle contraction in zebrafish embryos. Proceedings of the National Academy of Sciences of the United States of America 103 (2006), pp. 2713-8. 
633 Tao, Q., Yokota, C., Puck, H., Kofron, M., Birsoy, B., Yan, D., Asashima, M., Wylie, C.C., Lin, X. and Heasman, J. Maternal wnt11 activates the canonical wnt signaling pathway required for axis formation in Xenopus embryos. Cell $\mathbf{1 2 0}$ (2005), pp. 857-71.

637 638 S.S., Hansen, J.D., Winton, J.R., Bartholomew, J.L., Nagler, J.J., Walsh, P.J., Vijayan, M.M., Devlin, R.H., Hardy, R.W., Overturf, K.E., Young, W.P., Robison, B.D., Rexroad, C. and Palti, Y. Status and opportunities for genomics research with rainbow trout. Comp Biochem.Physiol B Biochem.Mol.Biol. 133 (2002), pp. 609-646.

Tipsmark, C.K. Identification of FXYD protein genes in a teleost: tissue-specific expression and response to salinity change. American journal of physiology. Regulatory, integrative and comparative physiology 294 (2008), pp. R136778.

Toro, S., Wegner, J., Muller, M., Westerfield, M. and Varga, Z.M. Identification of differentially expressed genes in the zebrafish hypothalamic-pituitary axis. Gene expression patterns : GEP 9 (2009), pp. 200-8.

Udvadia, A.J., Koster, R.W. and Skene, J.H. GAP-43 promoter elements in transgenic zebrafish reveal a difference in signals for axon growth during CNS development and regeneration. Development 128 (2001), pp. 1175-82.

Vallette-Kasic, S., Couture, C., Balsalobre, A., Gauthier, Y., Metherell, L., Dattani, M. and Drouin, J. The TPIT gene mutation M86R associated with isolated adrenocorticotropin deficiency interferes with protein: protein interactions. The Journal of clinical endocrinology and metabolism 92 (2007), pp. 3991-9. 
Vargas, A., Moreau, J., Landry, S., LeBellego, F., Toufaily, C., Rassart, E., Lafond, J. and Barbeau, B. Syncytin-2 plays an important role in the fusion of human trophoblast cells. Journal of Molecular Biology 392 (2009), pp. 301-18.

Volff, J.N. Turning junk into gold: domestication of transposable elements and the creation of new genes in eukaryotes. BioEssays : news and reviews in molecular, cellular and developmental biology 28 (2006), pp. 913-22.

Wilhelm, B.T. and Landry, J.R. RNA-Seq-quantitative measurement of expression through massively parallel RNA-sequencing. Methods 48 (2009), pp. 249-57.

Zhang, L., Kelley, J., Schmeisser, G., Kobayashi, Y.M. and Jones, L.R. Complex formation between junctin, triadin, calsequestrin, and the ryanodine receptor. Proteins of the cardiac junctional sarcoplasmic reticulum membrane. The Journal of biological chemistry 272 (1997), pp. 23389-97.

Zhou, Y.B., Cao, J.B., Yang, H.M., Zhu, H., Xu, Z.G., Wang, K.S., Zhang, X., Wang, Z.Q. and Han, Z.G. hZG16, a novel human secreted protein expressed in liver, was down-regulated in hepatocellular carcinoma. Biochemical and Biophysical Research Communications 355 (2007), pp. 679-86.

\section{Acknowledgments}

This work was supported by funds from the "Institut National de la Recherche Agronomique" (AIP bioressources INRA 2009) and by an EC's Seventh Framework Program (222719-LIFECYCLE). B.N. was supported by a joined Région Bretagne and INRA PhD fellowship. 
682 Figure 1 - The identification of rainbow trout genes that are predominantly 683 expressed in brain, testis, ovary, gill or muscle tissues.

684 Real-time PCR analysis was conducted using total RNA from the following tissues:

685 brain, pituitary, gill, heart, stomach, liver, intestine, muscle, skin, spleen, head kidney

686 (HK), trunk kidney (TK), oocyte, post-ovulatory ovary, and stage II testis. For each

687 tissue, three separate RT reactions were carried out using separate RNA samples that

688 originated from three different fish. The relative expression of each gene was

689 normalized to that of the $18 \mathrm{~S}$ gene and was calculated as the percentage of the highest

690 expression level recorded for each gene.

691 Figure 2 - The expression of $\beta$-enolase in muscle

692 Transverse sections through adult muscle showing a strong signal for the $\beta$-enolase

693 transcript in rainbow trout white fast muscle (WM). No signal is detected in red slow

694 muscle $(\mathrm{RM})$ or in the adipose tissue separating these two muscle regions.

695

696 Tables

697 Table 1 - Summary of the sequencing run results.

\begin{tabular}{|c|c|c|c|c|}
\hline Tissues & Number of reads & Number of base pairs & $\begin{array}{c}\text { Average sequence } \\
\text { length }\end{array}$ & $\begin{array}{c}\text { Number of runs } \\
\text { Gill }\end{array}$ \\
\hline Brain & 481.363 & 232.066 .637 & $378 \mathrm{bp}$ & 1 \\
\hline Ovary & 738.510 & 159.232 .104 & $331 \mathrm{bp}$ & 1.5 \\
\hline Testis & 1.234 .260 & 381.485 .303 & $318 \mathrm{bp}$ & 1 \\
\hline Total & $\mathbf{3 . 0 6 7 . 3 5 6}$ & $\mathbf{1 . 0 0 7 . 4 4 9 . 1 5 1}$ & $309 \mathrm{bp}$ & 1.5 \\
\hline
\end{tabular}


699 Table 2 - Selected genes that were over-expressed in the ovarian libraries.

700 The expression scores (representing the number of reads in the corresponding contig)

701 for the genes that are discussed within the text are shown for the ovarian and non-

702 ovarian libraries. Genes with expression profiles that were confirmed by qPCR are

703 identified in the table with an asterisk $(*)$ following the contig name.

\begin{tabular}{|l|l|l|l|}
\hline Contig name & Best Blast Hit & Ovarian & Non- \\
& & & ovarian \\
\hline BX082756.p.om.8 & ZPC domain containing protein 5 & 1042 & 1 \\
\hline CU072510.p.om.8 & ZPC domain containing protein 1 & 132 & 0 \\
\hline GAY7CUQ01AN5KC.p.om.8 & ZPA domain containing protein & 68 & 0 \\
\hline CB486811.p.om.8* & Protein Wnt-11 & 80 & 4 \\
\hline BX079798.p.om.8* & bucky ball & 87 & 0 \\
\hline BX074171.p.om.8 & oocyte protease inhibitor-2 & 248 & 2 \\
\hline CR373079.p.om.8 & oocyte protease inhibitor-2 (opi-2) & 170 & 2 \\
\hline BX303838.p.om.8 & serpin peptidase inhibitor & 169 & 0 \\
\hline CB490333.p.om.8* & POU domain, class 5, transcription factor 1 & 40 & 0 \\
\hline BX088048.p.om.8 & zygote arrest 1 (zar1) & 87 & 3 \\
\hline CX041779.p.om.8 & growth/differentiation factor 9 & 32 & 1 \\
\hline
\end{tabular}

Table 3 - Selected genes that were over-expressed in the testicular libraries.

706 The expression scores (representing the number of reads in the corresponding contig)

707 for the genes that are discussed within the text are shown for the testicular and non-

708 testicular libraries. Genes with expression profiles that were confirmed by qPCR are

709 identified in the table with an asterisk (*) following the contig name.

\begin{tabular}{|l|l|l|l|}
\hline Contig name & Best Blast Hit & Testicular & Non-testicular \\
\hline
\end{tabular}




\begin{tabular}{|c|c|c|c|}
\hline DQ489287.p.om.8 & GSDF & 2445 & 20 \\
\hline BX857318.p.om.8 & SYCP1 & 198 & 2 \\
\hline GBPNQUD01A6W4I.p.om.8* & $\begin{array}{l}\text { Myelin associated } \\
\text { glycoprotein }\end{array}$ & 372 & 1 \\
\hline CU063452.p.om.8 & $\mathrm{AMH}$ & 176 & 1 \\
\hline BX080625.p.om.8 & Cdk6 & 124 & 1 \\
\hline BX080735.p.om.8 & Cohesin subunit & 119 & 2 \\
\hline F0DLFKL01BENKG.p.om.8 & Unknown & 105 & 1 \\
\hline FYV3OTN02HHPOI.p.om.8 & Rec8 & 106 & 2 \\
\hline BX314487.p.om.8 & L-asparaginase & 85 & 1 \\
\hline FYV3OTN01B8PA8.p.om.8 & $\begin{array}{l}\text { ERV-FRD provirus } \\
\text { ancestral Env polyprotein }\end{array}$ & 68 & 2 \\
\hline F6VG15V02FTMKM.p.om.8 & $\begin{array}{l}\text { Histone-lysine N- } \\
\text { methyltransferase }\end{array}$ & 61 & 1 \\
\hline FYV3OTN01A1QIV.p.om.8 & $\begin{array}{l}\text { Zinc finger CW-type } \\
\text { PWWP domain protein } 1\end{array}$ & 53 & 1 \\
\hline CB487402.p.om.8 & LINE-1 reverse transcriptase & 62 & 5 \\
\hline FYV3OTN01E3SYW.p.om.8 & $\begin{array}{l}\text { Transposable element Tc1 } \\
\text { transposase }\end{array}$ & 47 & 1 \\
\hline F6VG15V02HO88H.p.om.8 & Pro-Pol polyprotein & 88 & 2 \\
\hline CX036404.p.om.8 & DAZL & 24 & 1 \\
\hline GBPNQUD01BLTP4.p.om.8* & TBX19 & 23 & 0 \\
\hline GBPNQUD01AIF47.p.om.8 & Retrotransposon protein 1 & 138 & 0 \\
\hline BX076816.p.om.8 & Envelope protein & 51 & 0 \\
\hline
\end{tabular}

711 Table 4 - Selected genes that were over-expressed in the muscle libraries.

712 The expression scores (representing the number of reads in the corresponding contig)

713 for the genes discussed within the text are shown for the muscle and non-muscle

714 libraries. Genes with expression profiles that were confirmed by qPCR are identified

715 in the table with an asterisk (*) following the contig name. 


\begin{tabular}{|l|l|l|l|}
\hline Contig name & Best Blast Hit & Muscle & Non-muscle \\
\hline CA343693.p.om.8* & Phosphoglycerate mutase 2-1 (muscle) & 5035 & 2 \\
\hline CR372691.p.om.8 & myosin, light polypeptide 3-3 & 2141 & 1 \\
\hline CA365356.p.om.8 & fast skeletal troponin C & 21213 & 10 \\
\hline CA345074.p.om.8* & Kelch repeat and BTB domain-containing protein 10 & 224 & 2 \\
\hline CA361268.p.om.8 & SET and MYND domain-containing protein 1 & 103 & 2 \\
\hline CX252008.p.om.8 & Calsequestrin-1 & 83 & 2 \\
\hline
\end{tabular}

717 Table 5 - Selected genes that were over-expressed in the brain libraries.

718 The expression scores (representing the number of reads in the corresponding contig)

719 for the genes that are discussed within the text are shown for the brain and non-brain

720 libraries. Genes with expression profiles that were confirmed by qPCR are identified

721 in the table with an asterisk (*) following the contig name.

\begin{tabular}{|l|l|l|l|}
\hline Contig name & Best Blast Hit & Brain & Non-brain \\
\hline BX082627.p.om.8* & BLBP & 1125 & 95 \\
\hline BX316810.p.om.8 & glial high affinity glutamate transporter & 261 & 1 \\
\hline CA368669.p.om.8 & Neuritin & 12 & 6 \\
\hline CA381682.p.om.8* & Neuromodulin & 176 & 2 \\
\hline CX153668.p.om.8 & Brain-specific polypeptide PEP-19 & 51 & 13 \\
\hline CA343423.p.om.8 & Synapsin & & \\
\hline
\end{tabular}

723 Table 6 - Selected genes that were over-expressed in the gill libraries.

724 The expression scores (representing the number of reads in the corresponding contig)

725 for the genes that are discussed within the text are shown for the gill and non-gill

726 libraries. Genes with expression profiles that were confirmed by qPCR are identified

727 in the table with an asterisk (*) following the contig name.

$$
\text { - } 32 \text { - }
$$




\begin{tabular}{|l|l|l|l|}
\hline Contig name & Best Blast Hit & Gill & Non-gill \\
\hline CA345680.p.om.8 & gill-specific calpain & 43 & 1 \\
\hline CA362824.p.om.8 & $\begin{array}{l}\text { Rh type C glycoprotein } \\
\text { (rhcg2) }\end{array}$ & 132 & 0 \\
\hline CA345079.p.om.8 & $\begin{array}{l}\text { Na/K ATPase alpha subunit } \\
\text { isoform 1a }\end{array}$ & 319 & 21 \\
\hline CA349792.p.om.8 & $\begin{array}{l}\text { FXYD domain containing } \\
\text { ion transport regulator 11 }\end{array}$ & 294 & 0 \\
\hline CA365840.p.om.8* & Envoplakin & 58 & 1 \\
\hline BX321817.p.om.8 & cornifelin-like protein b & 57 & 1 \\
\hline CA345392.p.om.8* & Zymogen granule membrane & 2075 & 10 \\
& protein 16 & & \\
\hline
\end{tabular}

\section{Additional files}

\section{Additional File 1 - Genes that were detected by Digital Differential Display} muscle and gill tissues.

733 This file contains the number of reads per condition for the contigs that were

734 identified to be predominantly expressed in rainbow trout ovary, testis, brain, muscle

735 and gill tissues (one folder page per tissue of interest) using the digital differential

736 display analysis. For each contig containing 10 or more reads in the tissue of interest

737 (FP in contig) and no hits in the other tissues (SP in contig), or for each contig having

738 a fold change of more than 10 (FP/SP), this file provides information on the

739 significance of the enrichment using Fisher's Exact Test (no value is given for contigs

740 with no hit in the SP condition). The file also provides information regarding the top

741 blast hit results for all of the contig sequences (best hit description, best hit database, 
742 the percentage of sequence coverage with the best hit, and the percentage of sequence

743 conservation with the best hit). For each contig, a dynamic internet link with the

744 corresponding contig view webpage is available (click directly on the contig name;

745 Excel file, .xls).

746 Additional File 2 - Primer sequences used for qPCR validation of some

747 differentially expressed genes.

748 